\title{
Ganglion cyst of the hoffa fat pad
}

Volume 9 Issue 2 - 2017

\section{Introduction}

Intra-articular knee masses are relatively rare clinical entities with a known differential diagnosis. The most common lesions are meniscal cysts, villous proliferation of the synovial membrane, Hoffa disease, lipoma, and ganglion cysts. This article presents a novel case of ganglion cyst of the Hoffa fat pad, which resolved spontaneously without any intervention.

\section{Case report}

A 28-year-old woman presented with right anterior knee pain for the past 4 months. The pain was localised to the inferolateral aspect of the knee joint and came only at the extremes of knee movement. The pain was worse at night, though she hadn't appreciated early morning stiffness or improvement with activity. There were no mechanical symptoms such as locking, catching, or instability of the knee.

On physical examination, the patient had no effusion in the knee. Range of motion of the right knee was from $0^{\circ}$ to $125^{\circ}$ with pain at the extreme of flexion. There was full extension and flexion to $135^{\circ}$ in the contralateral left knee. Palpation of the joint line elicited no tenderness or palpable masses. There was no varus or valgus instability of the right knee. Anterior and posterior drawer tests were negative. The Lachman's test was also negative. Neurologic examination of the lower extremity revealed no deficits and the dorsalis pedis and posterior tibialis pulses were symmetric in both lower extremities.

Radiographs obtained to evaluate the patient's knee pain were normal. Based on her symptoms, magnetic resonance imaging (MRI) scan of her right knee was advised.

The MRi findings revealed a homogenous fluid intensity cystic lesion within the Hoffa's fat pad. STIR axial \& sagittal images showed well defined fluid signal intensity lesion in Hoffa's fat pad. Infrapatellar fat is suppressed on STIR image, making fluid signal to appear bright (Figure 1a \& 1b). The differential diagnosis and management of infrapatellar lesions of the knee are discussed.

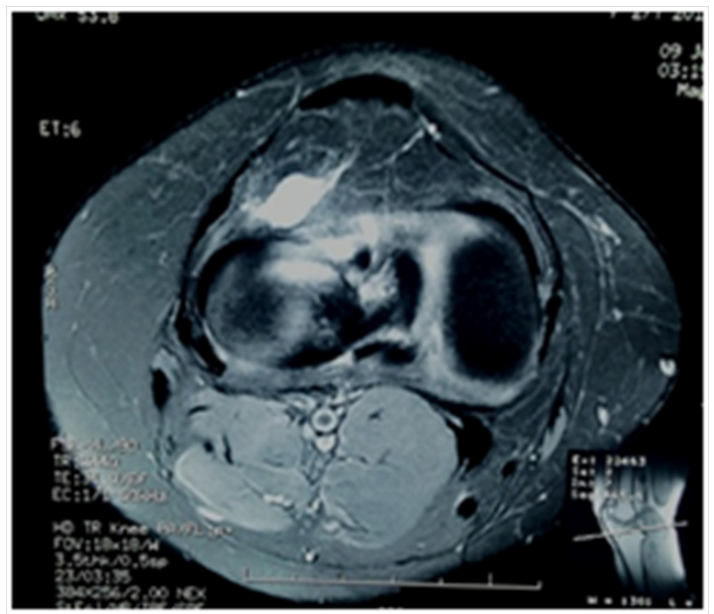

Latika Gupta, ${ }^{2}$ Tushant Kumar, ${ }^{2}$ Anil Rawat,' Vikas Agarwal'

'Department of Clinical Immunology, Sanjay Gandhi

Postgraduate Institute of Medical Sciences, India

${ }^{2}$ Department of Radiodiagnosis, Dr. Ram Manohar Lohia Institute of Medical Sciences, India

Correspondence: Vikas agarwal, Professor, Department of Clinical Immunology, Sanjay Gandhi Postgraduate Institute of Medical Sciences, Lucknow, India, 2260I4, Tel 522-24943I8, Email vikasagr@ahoo.com

Received: October 31, 2016 | Published: October 20, 2017

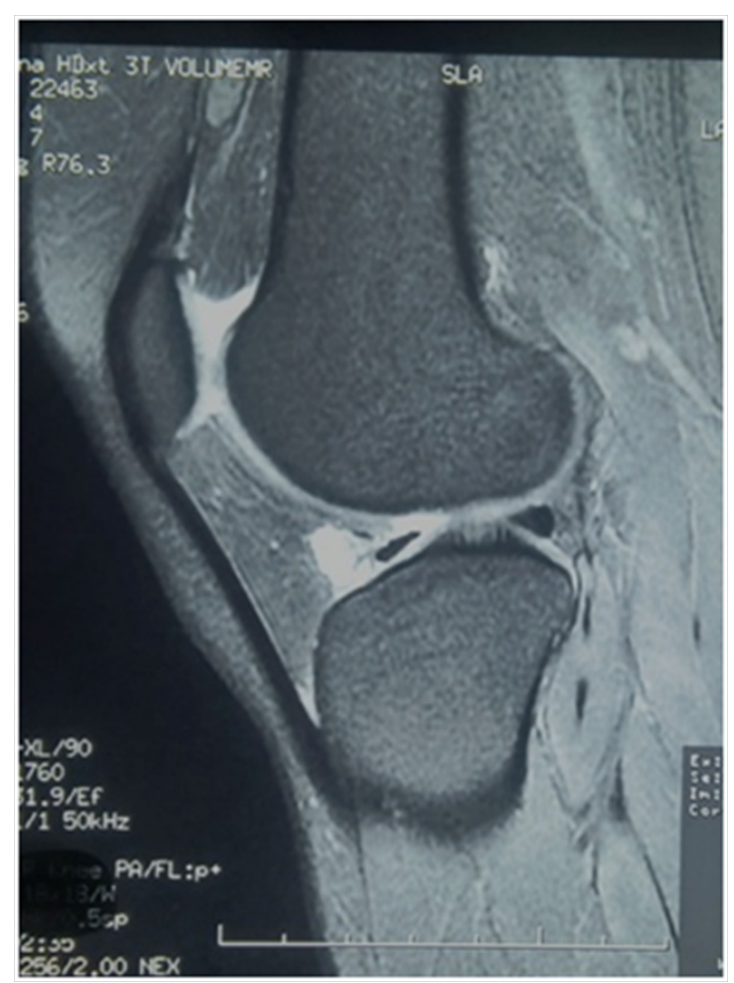

Figure Ia\& I b Axial \& sagittal STIR MRI images showed a well-defined $2 * 2$ $\mathrm{cm}$ fluid intensity cystic lesion in the Hoffa fat pad close to the anterior horn of the lateral meniscus (White arrow).

\section{Discussion}

Intra-articular ganglion cysts are rare. Currently, two theories relating to the pathogenesis of ganglion cysts exist, although the true cause is still unknown. ${ }^{1,2}$ The first theory attributes the presence of ganglion cysts to being a product of mucinous degeneration of 
the connective tissue. ${ }^{3,4}$ The second theory considers it a cause of herniation of synovial tissue through a defect in the joint capsule or tendon sheath, similar to those of wrist joint origin. ${ }^{2,4}$ For both theories, the relationship to previous trauma is uncertain and has not been documented.

Ganglion cysts contain thick, sticky, clear, colourless, jellylike material. These cysts, when associated with an adjacent ligament, are associated with mucoid degeneration of the ligament with degenerative cysts at its attachment. ${ }^{2}$ Long-standing pressure of a ganglion cyst against an adjacent hard surface may produce an indentation through gradual erosion of the bone, creating an intraosseous ganglion. The high incidence of intraosseous ganglia in patients with either mucoid degeneration of the cruciate ligament or intrasubstance cruciate ligament ganglia suggests that these two entities may share a similar pathogenesis.

Cysts in the knee are often associated with meniscal tears, providing a means for synovial fluid to communicate with the lesion, more so in the region of the lateral meniscus. However, lesions have been described even in the absence of ligamentous pathology, as in our case. ${ }^{5}$

Ganglion cysts are usually asymptomatic, and are often hard to diagnose clinically due to the lack of specific symptoms and signs. Symptomatic patients may present with pain aggravated with flexion or activity, increased pain during posture change, locking, clicking or popping sensations, and a decreased range of motion. Because the Hoffa fat pad is relatively spacious, it may take time for the mass to develop into a size large enough to cause symptoms.

Ganglion cysts are identifiable on MRI as isointense to fluid with low TI-weighted signal intensity and high T2-weighted signal intensity. Lipomas in the Hoffa fat pad; albeit rare, are more common than ganglion cysts, and often present similarly, hence MRI is the only non-invasive test that can differentiate them. A simple lipoma is uniformly isointense to fat on all MRI sequences and looks hypointense on fat saturation sequences. ${ }^{6}$

Similar to lipomas, lipoma arborescens may present with slow enlargement of a painless mass accompanied by intermittent effusions. These lesions, however, have villous projections of fatty synovial tissue. Magnetic resonance imaging usually reveals a synovial mass with a frond-like architecture, fat-signal intensity on all pulse sequences and suppression of signal with fat-selective presaturation. This again differs from the clear margins of a ganglion cyst. $^{7}$

Hoffa disease is a syndrome of infrapatellar fat pad impingement resulting from inflammation of the fat. It usually begins with minor trauma, which may go unnoticed, resulting in hemorrhage into the fat pad. This stimulates hypertrophy and inflammation in the region, resulting in further impingement due to lack of adequate space in the tight compartment. ${ }^{8}$ Sometimes chondroid metaplasia can occur and these are then called as synovial chondromas. In the subacute and chronic phases, fibrin and hemosiderin can develop, and are easily identifiable by different picture on MRI, with blooming artifact seen due to hemosiderin deposits. It is only once fibrous tissue ossifies, that the lesion is visible on plain radiographs. This patient had no history of trauma and no ossification could be seen on radiographs.

Histopathologic examination after arthroscopic or open cysts excision is the gold standard for establishing the diagnosis in all cystic lesions of the knee. However, since symptoms are minimal, very few patients are willing to undergo aspiration or excision.

Arthroscopic resection is the treatment of choice for ganglion cysts in relation to the knee. In the absence of an associated tear in the menisci, ganglion cysts may not be appreciated at arthroscopy and may require an extra-articular rather than an arthroscopic approach for decompression. For extraarticular lesions, sonographically guided aspiration can be attempted, though recurrences can occur. ${ }^{9}$

The patient wasn't willing for arthroscopic surgery in the present case, hence ultrasound guided cyst aspiration was advised. Interestingly, pain subsided spontaneously a month after diagnosis, obviating the need for intervention. Sonogram of the knee at that time showed normal anatomy with absence of any cystic lesion, suggesting spontaneous rupture and resolution. There was no history of trauma or intense exertion during this period.

Although MRI is the ideal investigation for imaging the cystic lesions in the knee, the lesion in our case was large and superficial and unlikely to be missed on ultrasound. Since MRI is an expensive investigation, it was deemed unnecessary in the absence of symptoms. The patient was asymptomatic on three-year follow up, essentially ruling out the possibility of a residual cyst. To our knowledge, this is the first reported case with spontaneous resolution. This highlights that watchful waiting for a while may be advisable in these cases, before embarking on invasive procedures.

\section{Acknowledgments}

None.

\section{Conflicts of interest}

None.

\section{References}

1. Kumar A, Bickerstaff DR, Grimwood JS, et al. Mucoid cystic degeneration of the cruciate ligament. J Bone Jt Surg. 1999;81:304-305.

2. Kaatee R, Kjartansson O, Brekkan A. Intraarticular Ganglion between the Cruciate Ligaments of the Knee: A Case Report. Acta Radiol. 1994;35(5):434-436.

3. Fealy S, Kenter K, Dines JS, et al. Mucoid degeneration of the anterior cruciate ligament. Arthrosc J Arthrosc Relat Surg. 2001;17:1-4.

4. Angelides AC, Wallace PF. The dorsal ganglion of the wrist: Its pathogenesis, gross and microscopic anatomy, and surgical treatment. $J$ Hand Surg. 19746;1(3):228-235.

5. Stein D, Cantlon M, Mackay B, et al. Cysts about the knee: evaluation and management. J Am Acad Orthop Surg. 2013;21(8):469-479.

6. Jacobson JA, Lenchik L, Ruhoy MK, et al. MR imaging of the infrapatellar fat pad of Hoffa. Radiogr Rev Publ Radiol Soc N Am Inc. 1997;17:675-691.

7. Kataria H, Kapoor SK, Patra SR, et al. Lipoma arborescence of the knee in a child - a diagnostic dilemma: radiological and arthroscopic evaluation. J Orthop Sci Off J Jpn Orthop Assoc. 2010;15:414-419.

8. Eymard F, Chevalier X. Inflammation of the infrapatellar fat pad. $J t$ Bone Spine Rev Rhum. 2016;83:389-393.

9. Ju BL, Weber KL, Khoury V. Ultrasound-Guided Therapy for Knee and Foot Ganglion Cysts. J Foot Ankle Surg. 2016;56(1):153-157. 